\title{
Cross-sectional Diameter
}

National Cancer Institute

\section{Source}

National Cancer Institute. Cross-sectional Diameter. NCI Thesaurus. Code C139032.

A measurement of a structure taken along the plane that is perpendicular to the long axis. 